\title{
Magneto-active Slosh Control Experiment
}

\author{
Cassandra Bossong, Liam Carls \\ Department of Physics \& Astronomy, Carthage College \\ Kenosha, WI, United States
}

\begin{abstract}
The Magneto-active Slosh Control (MaSC) project seeks to demonstrate active slosh control in cylindrical propellant tanks by means of a thin, flexible metallic membrane that is acted on by a magnetic field in order to reposition itself to produce an optimized force on the surface of the liquid. Slosh is a destabilizing effect of engine burns and other orbital operations. The mitigation of slosh is a mission-critical effort in the aerospace industry. The overall objectives for the Magneto-active Slosh Control experiment are to demonstrate slosh control forces on liquid surfaces exerted by a high-permeability magnetic membrane in an external magnetic field, as well as develop active sensing and control of the membrane's position in microgravity environments.
\end{abstract}

\section{Introduction}

Unintended motion of propellant has caused countless negative effects on spacecraft, ranging from instability to unexpected explosions. Fuel mass gauging is confounded by liquid sloshing in propellant tanks. Liquid sloshing can potentially cause destabilization of the spacecraft, resulting in catastrophic failures. When sloshing occurs, it changes the configuration of the liquid, which greatly affects the center of mass of the vehicle. In addition to this, sloshing can also cause the fluid to be vented in space, rather than the gas, which would waste the fluid and potentially cause performance issues on the spacecraft due to various thermodynamic effects. The bigger the tank, the longer it takes the tank to equilibrate, thus producing more slosh inside the tank for a longer period of time. For example, Space X's Falcon 1 failed several times due to liquid-oxygen sloshing in the second stage of the vehicle. Currently, liquid sloshing remedies include baffles on the inner walls of the propellant tanks, or an elastic membrane on the surface of the fluid. The Magneto-active Slosh Control (MaSC) project brings together two existing efforts in the space industry, active slosh suppression and propellant gauging in low gravity environments. This project is a collaboration with Embry-Riddle Aeronautical University to develop a free-floating metallic membrane that is autonomously controlled in order to suppress slosh forces in propellant tanks. This technique would use less mass and less power than the current technologies to prevent sloshing in propellant tanks. This is important because every piece of technology in the spacecraft costs something, whether that be crew safety, range of the mission, or carrying less fuel to take in account for something else.

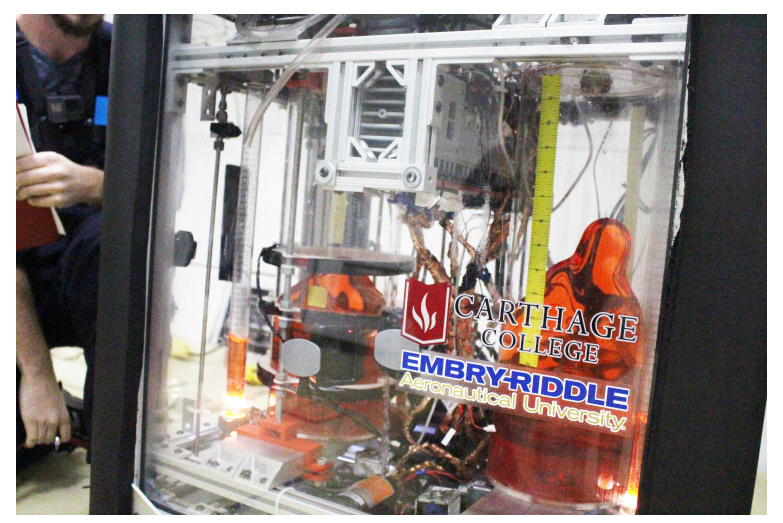

Figure 1: Liquid behavior in low gravity on the MaSC experiment from November 2019. Note that the fluid is suppressed in its cylindrical container at the lower left in the figure. 
1.1. Purpose The development of the MaSC technology would address several limitations of current slosh suppression systems. The first anti-slosh remedy is baffles on the inner walls of the propellant tank, typically one that is cylindrical. The baffles provide a significant damping effect on the slosh, however this extra material adds to the mass of the overall structure of the tank. Essentially, the baffles add mass of the tank, which reduces the volume of propellant that can be stored in the tank. The other slosh suppression method is an elastic membrane, which is a thin polymer structure that is located inside the tank, typically on the surface of the fluid. This method of suppressing slosh also comes with restrictions that include the elastic coefficient of the membrane not being high enough to withstand the sloshing as well as the fact that this type of membrane can only be effectively used on a spherical tank, which is uncommon in spacecraft vehicles. Although these methods have proven to dampen sloshing in propellant tanks, the Magneto-active Slosh Control (MaSC) approach would be more effective and cost-efficient. This technique uses the concept of a free-floating membrane to suppress slosh in propellant tanks. The membrane is a thin magnetic alloy which is actively and autonomously controlled to suppress slosh forces occurring during engine burns and other spacecraft maneuvers.

1.2. Collaboration with Embry-Riddle The Magneto-active Slosh Control project is a joint-effort between the Carthage Space Sciences Program and Embry Riddle Aeronautical University to develop a free-floating membrane to suppress slosh in propellant tanks. The general concept behind this experiment was a magneto-active propellant management device, or a MAPMD. This approach was patented by Embry-Riddle, which sparked the use of a metallic membrane, in this case, metglas. In collaboration with Embry-Riddle, Carthage College has added active control of the membrane in low gravity as well as understood the physics behind it. In zero gravity, the position of the membrane can be directed by exerting forces on it magnetically so essentially we are able to detect the presence of the membrane and reposition it using coils.

1.3. Concept The concept behind the MaSC experiment will essentially reduce the overall structural mass of the propellant tank. It will also be able to dampen sloshing tendencies while providing real-time position tracking on the location of the fluid in the cylindrical tank. Carthage College and Embry-Riddle Aeronautical University are working together to develop the various elements of the project: the metallic alloy membrane made out of metglas, the coil system located outside of the experimental tank that will attempt to magnetize the metallic membrane, and the "field gradient control" software that will provide a strategic current profile to the coil system in order to produce an optimized force on the membrane.

\section{Parabolic Flight Campaign}

2.1. Microgravity Team Undergraduate students at Carthage College with diverse backgrounds have worked together to develop the Modal Propellant Gauging (MPG) technology, a vibration-based fuel gauging system suitable for operation low gravity since 2008. The past few years have also been dedicated to the Magneto-active Slosh Control (MaSC) experiment, which brings together two existing efforts of fluid physics, including propellant gauging as well as active slosh suppression in low gravity environments. Twice a year, the microgravity students fly with their payload and their advisor, Dr. Kevin Crosby, on parabolic flights through the Zero-G Corporation, previously with NASA until their program was discontinued. The parabolic flight campaign is funded through NASA's Flight Opportunities Program. NASA has also funded multiple iterations of the MPG project, all of which were advised by Dr. Crosby. These projects give students valuable hands on experience with space technology development through partnership with NASA and other academic researchers around the world. It is essentially graduate level research in an undergraduate setting.

2.2. Zero G Corporation The Carthage College Microgravity team takes part in a parabolic flight campaign in partnership with The Zero G corporation. These flights take place in Florida, twice a year on a modified Boeing 727 called G-Force One. The inside of the plane is emptied out and padded in order to implement the different experiments from various universities, including one from Carthage College. The plane flies in a parabolic trajectory, which allows the researchers and payload to experience "Zero Gravity" as if they were on the International Space Station, as shown in Figure 2. There are roughly 30 parabolas on these research flights, giving the experiments and the researchers on board around 10 minutes total in zero gravity in increments of 22 seconds at a time. The MaSC experiment flew with Zero G in November of 2020, along with another Carthage payload. 


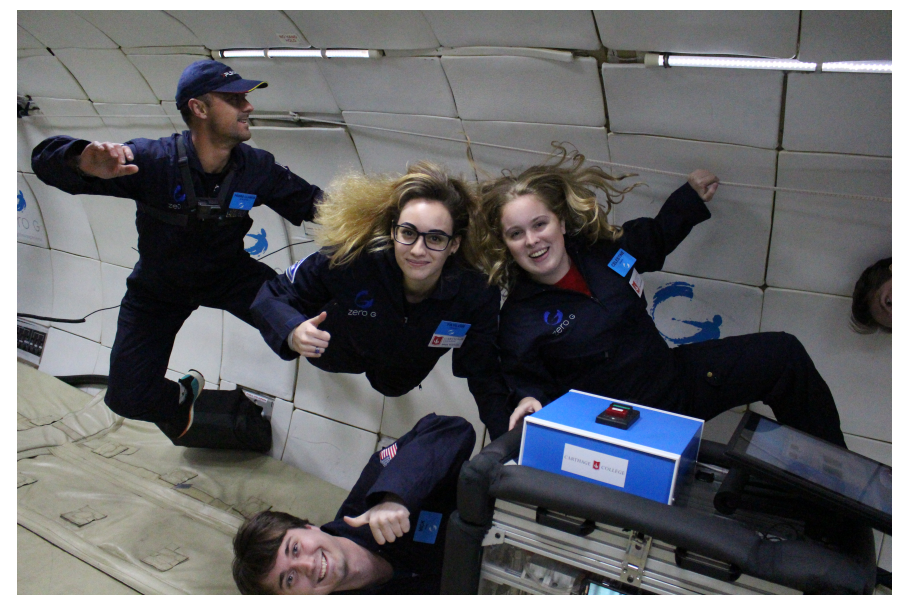

Figure 2: Carthage students and Embry-Riddle professor on board G-Force One during a "Zero-G" parabola in Nov. 2019.

\section{Experimental Flight Payload}

3.1. Payload Design The heart of the MaSC experiment is designated by two 7.7 liter polycarbonate tanks. These tanks are part of a flow loop, which consists of all components and interfaces that hold or transfer liquid, in this case water. The experimental tank (Tank A) has three small coils of wire on the exterior diameter to produce a magnetic effect on the metallic membrane inside the tank. However, the control tank (Tank B) has no coils or magnetic ring, and is just used for comparison purposes during the data analysis portion of the project. The flow loop includes six solenoid valves, two pumps, and other components housed in the secondary containment vessel. A full diagram of the flow loop, which resides inside secondary containment, is shown in Figure 3. The valves are manually controlled through interfacing with an advanced computer software on a monitor situated on the top of the payload. The software is called LabVIEW, which is a system-design platform that is programmed to run a specific environment which is compatible with our payload design and data acquisition system. LabVIEW allows us to operate the flow loop, as well as control the programmable power supply, which is important for setting the current of each coil during flight.

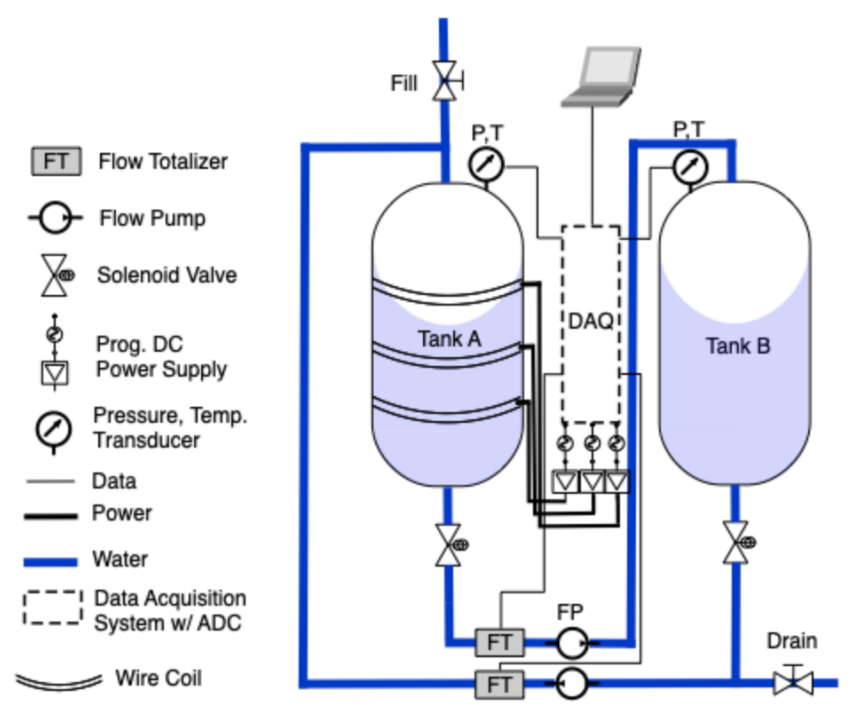

Figure 3: Overview of Flow Loop and Data Acquisition System. 
3.2. Electromagnetic Description Magnetic fields are generated by moving charges and current in a wire; creating coils by winding wire in a circular loop allows us to create a controlled external magnetic field. The strength of the external field relies on the radius of the coil itself, the number of turns of wire, in this case 100 turns, and the current amperage. The magnetic field generated by these coils are non-divergent, so essentially the field will wrap back around to where the field originally started, as shown in Figure 4.

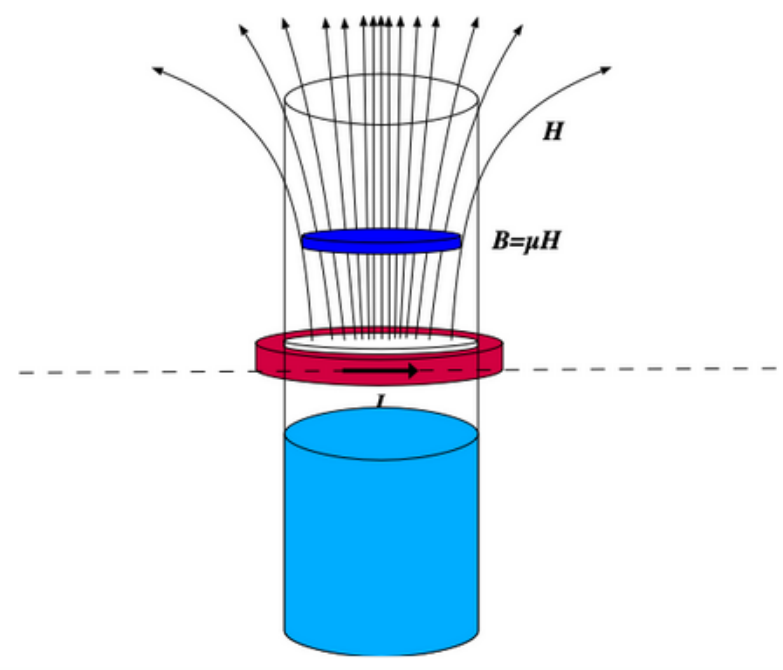

Figure 4: Magnetic field generated by the coil interacting with the membrane installed in the experimental tank.

The equation for the magnetic field (B) is shown in Equation 1, typically measured in Gauss (G). R represents the radius of the coil, $\mathrm{N}$ is the number of turns in the coil, I is the current, and $\mu$ is the magnetic permeability of free space. The $\mathrm{z}$ component represents the distance away from the coil along the $\mathrm{z}$-axis, which is important for the magnetic force. The relationship between the coils and the membrane will generate a force downwards, thus reducing slosh on the surface of the liquid in the tank.

$$
B_{z}=\frac{\mu I R^{2}}{2}\left(R^{2}+z^{2}\right)^{\frac{-3}{2}}
$$

Equation 1: The magnetic field of a coil in the $\mathrm{z}$ direction.

3.3. Analytical Model A magnetic field surrounding two coils can produce varying configurations depending on the direction of the current. The magnetic field is calculated in Equation 1 for the field everywhere in space near a coil of current. From this equation, we can use the Generalized Lorentz Force to determine the force acting on the membrane sitting in the magnetic field, however we only want to focus on the z-component in regards to the force. The z-component of the force on an object is proportional to the field multiplied by the derivative of the field along the z-axis, which is shown in Equation 2, and represented graphically by Figure 5. This allows us to control the force by varying the current ratio between the top and bottom coils, which will help us find the optimal location for the membrane to exert a restoring force on the liquid's surface.

$$
F \propto B \frac{d B}{d z}
$$

Equation 2: The proportionality of force and magnetic fields. 


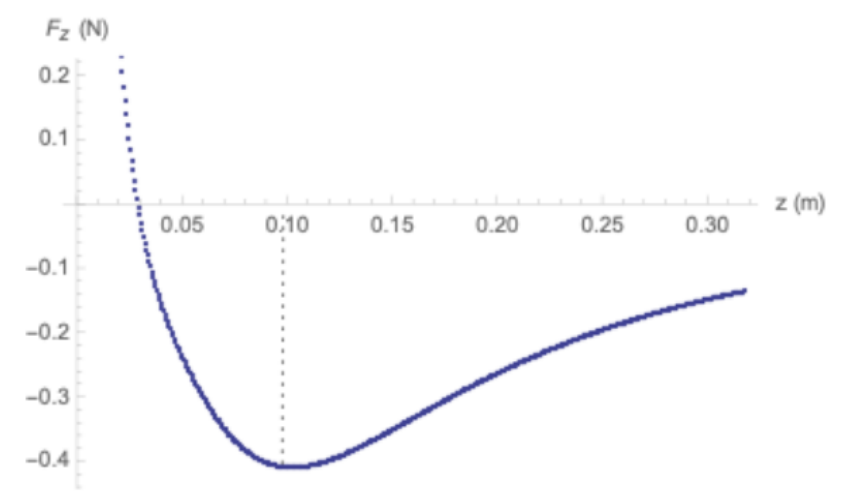

Figure 5: Graphical representation of the relationship between the force and z-component of the magnetic field.

\section{Data Analysis}

For the MaSC experiment, the primary objectives were to develop active sensing and control of the high permeability membrane in microgravity to produce a force on the surface of the liquid to prevent sloshing. In order to determine the position of the membrane while actively demonstrating a force on the liquid's surface, we decided to use a feature recognition software called OpenCV. Each membrane was painted with colored squares, as seen in Figure 6, in order to make it easier to track their position during the zero gravity portion of the flight. The image in Figure 6 shows the exact angle at which the data will be analyzed after obtaining the GoPro footage from the entire flight. The membrane has a distinct background to provide enough contrast for not only the Go-Pros to distinguish but also the feature recognition software. The software will attempt to track the position of a square on the membrane using the GoPro footage obtained from flight week. From there, it will take this data and convert it to a .csv file for further analysis. The MaSC project flew on a parabolic flight campaign in November 2020.

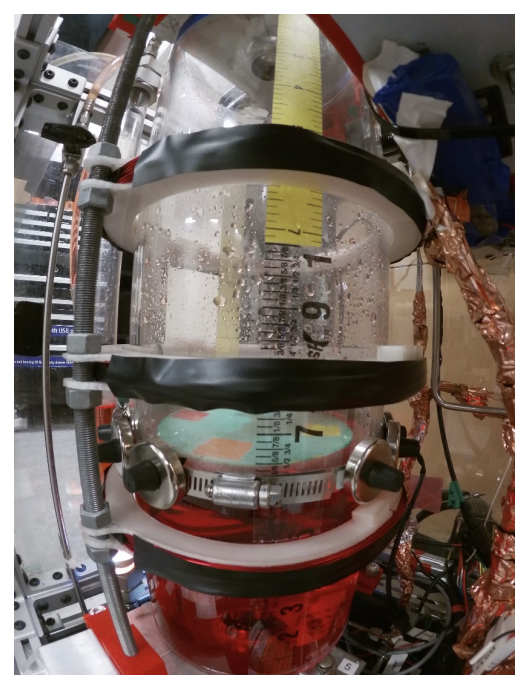

Figure 6: Still shot of the GoPro view angle of the experimental tank. Note the different colored squares on the visible surface of the membrane used for tracking its motion.

\section{Summary}

The MaSC experiment is a joint-effort between Carthage Space Sciences and Embry-Riddle Aeronautical University for the purpose of developing active sensing and control of a high permeability membrane in low-gravity environments, while producing a force on the surface of the liquid to prevent sloshing. This method of slosh suppression would be 
more effective and cost-efficient than current slosh dampening remedies. Reducing slosh is mission critical in the aerospace industry because of the significant negative effects sloshing has on spacecraft and other vessels. The MaSC payload flew on a parabolic flight campaign in November 2020, providing crucial data that will prove its effectiveness in the aerospace industries.

\section{Acknowledgments}

We would like to thank our advisor, Dr. Kevin Crosby, for giving us these amazing opportunities to enhance our undergraduate careers. We would additionally like to thank the Wisconsin Space Grant Consortium for funding this project as well as our collaborators at Embry-Riddle Aeronautical University for playing a significant role in the experiment's success. Finally, we would like to thank the Zero-G corporation for allowing us the opportunity and the unforgettable experience to test our project in low gravity environments.

\section{References}

Sivasubramanian et al (11 September 2018). Hybrid Magneto-Active Propellant Management Device for Active Slosh Damping within a Vehicle Fuel Tank. US 10,071,825 B2, United States Patent.

Storey, Jedediah (18 Dec. 2014). "Experimental, Numerical and Analytical Characterization of Slosh Dynamics Applied to In-Space Propellant Storage, Management, and Transfer." NASA, National Aeronautics and Space Administration, www.nasa.gov/content/experimental-numerical-and-analytical-characterization-of-slosh-dynamics-appliedto-in-space/.

Wade, Mark. (2019) Falcon 1, Astronautix,www.astronautix.com/flfalcon1.html. 